\title{
Efficacy of Hyperbaric Oxygen Therapy on Central Corneal Thickness, Intraocular Pressure, and Nerve Fiber Layer in Patients with Type 2 Diabetes: A Prospective Cohort Study
}

\author{
Havva Kaldırım¹, Kürsat Atalay ${ }^{1}$, Banu Ceylan² ${ }^{2}$ Serpil Yazgan ${ }^{3}$ \\ ${ }^{1}$ Department of Ophthalmology, Bagcilar Training and Research Hospital, Health Sciences University, Istanbul, Turkey \\ ${ }^{2}$ Department of Underwater and Hyperbaric Medicine, Bagcilar Training and Research Hospital, Health Sciences University Istanbul, \\ Turkey \\ ${ }^{3}$ Department of Ophthalmology, Medical Faculty, Inonu University, Malatya, Turkey
}

Purpose: To evaluate the effect of hyperbaric oxygen therapy (HBOT) on central corneal thickness (CCT), intraocular pressure (IOP), and the retinal nerve fiber layer (RNFL) thickness in patients with type 2 diabetes mellitus.

Methods: This prospective non-randomized cohort study consisted of type 2 diabetes mellitus patients who received 30 sessions of HBOT for diabetic foot ulcer. The CCT, IOP, and RNFL measured at baseline, after the 10th session of HBOT, after the 20th session of HBOT, after the 30th session of HBOT, and after the 3 months of the last session of HBOT. We gained the superior-nasal, superior-temporal, inferior-nasal, inferior-temporal, nasal and temporal quadrant RNFL values with a spectral-domain optical coharence tomography.

Results: Forty-six eyes of 46 patients included in the study. During the study period, a statistically significant increase in mean IOP values compared to baseline was observed $(p<0.001)$. We found no significant changes at CCT and all quadrants of RNFL values during HBOT and after 3 months of the treatment $(p>0.05)$. During the study period, the IOP levels increased over $21 \mathrm{mmHg}$ (between 22 and $28 \mathrm{mmHg}$ ) in seven eyes (15.2\%). The mean hemoglobin A1c values of these patients with IOP $>21 \mathrm{mmHg}$ were $8.2 \pm 0.9 \mathrm{mg} / \mathrm{dL}$, and there was significant differences compared with those of patients with IOP values $\leq 21$ $\mathrm{mmHg}(7.4 \pm 2.8 \mathrm{mg} / \mathrm{dL})(p=0.001)$.

Conclusions: HBOT increase IOP in type 2 diabetic patients especially in ones with impaired blood glucose regulation. However, it does not cause any changes in CCT and RNFL. As diabetic retinopathy and diabetic foot ulcer are in common pathologies, thus this brief report concludes a need for further studies with longer follow-up periods to explore the potential interaction of HBOT on CCT, IOP, and RNFL.

Key Words: Central corneal thickness, Hyperbaric oxygenation, Intraocular pressure, Retinal nerve fiber layer, Type 2 diabetes mellitus

Received: May 27, 2020 Final revision: October 12, 2020 Accepted: October 19, 2020

Corresponding Author: Havva Kaldırım, MD, FEBO. Department of Ophthalmology, Bagcilar Training and Research Hospital, Health Sciences University, Merkez Mahallesi Dr. Sadık AHMET Caddesi BAĞCILAR, Istanbul, Turkey. Tel: 90-212-440-4000, Fax: 90-212-440-4242, E-mail: istanbuleah30@ saglik.gov.tr

This study was presented at oral presentation section of 15th International Symposium in Ocular Pharmacology and Therapeutics on November 7-9, 2019 in Valencia, Spain. 
Hyperbaric oxygen therapy (HBOT) is effective and noninvasive treatment that has been applied to treat various conditions related to hypoxia, including decompression sickness, acute carbonmonoxide intoxication, air embolism, soft tissue infections, radiation necrosis, and impaired wound healing [1].

HBOT increases oxygen pressure and concentration of reactive oxygen species (ROS) in tissue [2]. An excess of ROS in tissues and/or deficiencies in antioxidant activity, may contribute to complications of HBOT [3,4]. The eye, with its intense exposure to light, robust metabolic activity and in certain regions, high oxygen tension, is particularly susceptible to oxidative damage. Reactive oxygen and nitrogen species have been implicated in a growing number of medical conditions affecting the human eye. For example, ROS and deficiencies in antioxidant activities contribute to the pathogenetic processes in cataract, age-related macular degeneration and keratoconus [5-8].

ROS can play a role in the pathogenesis of glaucoma by stimulating apoptosis and inflammatory pathways on the level of the trabecular meshwork (TM) [9]. The exact molecular impact of ROS on the TM is uncertain, although small studies have suggested reduced cellular adhesion to the surrounding extracellular matrix, overexpression of extracellular matrix proteins such as fibronectin leading to reduced TM-cell permeability and direct damage as key mechanisms [10-12].

Our aim to evaluate the effect of HBOT on central corneal thickness (CCT), intraocular pressure (IOP), and retinal nerve fiber layer (RNFL) thickness in patients with type 2 diabetes mellitus (DM).

\section{Materials and Methods}

This prospective, nonrandomized-cohort study was conducted at Department of Retina, University of Health Sciences between June 2018 and June 2019. Ethical approval was obtained from the Local Research Ethics Committee (project no. 2017.12.1.04.020), and the study performed according to the tenets of the Declaration of Helsinki. The nature and purpose of the study were explained in detail to all participants, and written informed consent was obtained.

Forty-six eyes of 46 consecutive patients with a diagnosis of diabetic foot ulcer (DFU) and planned to 30 sessions
HBOT were included in this study. Participant's right eyes were evaluated. Patients who undergoing argon laser photocoagulation, ocular surgery and antivascular endothelial growth factor therapy in less than 1 years, have uncontrolled systemic or ocular diseases, consuming alcohol and tobacco, refractive error beyond -2.0 and +2.0 diopters, corneal diseases, signs or history of glaucoma or ocular hypertension, neovascularization or edema on optic disc, optic disc anomalies and noncompliant patients were also excluded the study.

\section{HBOT procedure}

HBOT was carried out in a multiplace hyperbaric chamber once a day (between 8:30 a.m. and 10:00 a.m.), five times a week. The treatment protocol consisted of 120 minutes lasting periods of inhalation of $100 \%$ oxygen at a pressure of 2.5 atmospheres absolute, interspersed with two "air breaks" lasting 5 minutes, after each 30 minutes of inhalation. All of the patients underwent 30 sessions of HBOT.

\section{Ophthalmological evaluation}

Demographic data from those involved in the study were obtained baseline and a complete ophthalmologic examination including assessment of best-corrected visual acuity, measurement of IOP, slit lamp biomicroscopy and dilated fundus examination were performed. Fundus florecein angiography (FFA) imaging and anterior chamber angle examination with the Goldman three-mirror contact lens were done all patients at baseline and after three months of last session of HBOT.

CCT was measured by the same ophthalmologist using Lenstar LS 900 (Haag-Streit AG, Köniz, Switzerland). After instillation of a mixture of $0.5 \%$ proparacaine and fluorescein into the eye, IOP was measured by Goldmann applanation tonometry and the average of three measurements was recorded. IOP measurements of the patients were made between 10:00 a.m. and 10:30 a.m. during the study. Peripapillary RNFL thickness was measured by automatically using a spectral-domain optical coherence tomography device (Spectralis; Heidelberg Engineering, Heidelberg, Germany). All of the spectral-domain optical coherence tomography images had a signal strength of 9 or better. For RNFL analysis, a 3.45-mm diameter peripapil- 
lary ring was measured as six quadrants containing nasal-superior (NS), temporal-superior (TS), nasal-inferior $(\mathrm{NI})$, temporal-inferior $(\mathrm{TI})$, nasal $(\mathrm{N})$ and temporal $(\mathrm{T})$. All measurements were obtained at baseline (CCT-b, IOP-b, and RNFL-b), after 10th session of HBOT (CCT-1, IOP-1, and RNLF-1), after 20th session of HBOT (CCT-2, IOP-2, and RNLF-2), after 30th session of HBOT (CCT-3, IOP-3, and RNLF-3) and after 3 months last session of HBOT (CCT-4, IOP-4, and RNLF-4). Hemoglobin A1c (HbAlc) (\%) values were gained before of HBOT.

\section{Statistical analysis}

Statistical analysis of the study was done by using IBM SPSS Statistics ver. 19.0 (IBM Corp., Armonk, NY, USA). The distribution of data was determined with a Shapiro-Wilk test. The descriptive statistics of continuous variables were expressed as mean \pm standard deviation and categorical variables were expressed as frequency and percent. Friedman's ANOVA test was used for the comparison of variables of measurements at different times. Paired Samples $t$-test or Wilcoxon sign rank test were used to compare baseline and each measured value of different times according to the distribution of data. Kruskal-Wallis test was used to compare groups created according to HbAlc values. A $p$-value of less than 0.05 was considered statistically significant in all test except for Friedman's ANOVA test $(p<0.01$ was considered statistically significant).

\section{Results}

This study included 46 eyes of 46 type 2 diabeticpatients who have DFUs. Thirty-three patients were male and 13 patients were female. The median age was $58.58 \pm 3.30$ years (range, 53-65 years) and duration of diabetes was $20.52 \pm 2.84$ years (range, 16-27 years). The mean visual acuity during study period was $0.22 \pm 0.13 \log$ MAR (between 0.3 and $0.0 \log$ MAR). All patients were phakic. The average cup-to-disc ratio in the study subjects was between 0.1 and 0.3 and absence of glaucomatous disc appearance. According to the baseline FFA findings, 17 eyes have mild-moderate non-proliferative DRP, 11 eyes have severe non-proliferative diabetic retinopathy (DRP) and 18 eyes have no proliferative diabetic retinopathy findings due to applied laser photocoagulation at least 1 years ago.
Table 1. Comparison of IOP and CCT values with baseline and consecutive measurement values after HBOT

\begin{tabular}{lcl}
\hline Parameter & Value & $p$-value \\
\hline IOP $(\mathrm{mmHg})$ & & \\
IOP-b & $16.17 \pm 2.71$ & NA \\
IOP-1 & $18.15 \pm 3.44$ & $p_{1}<0.001^{*}$ \\
IOP-2 & $18.52 \pm 4.03$ & $p_{2}<0.001^{*}$ \\
IOP-3 & $18.39 \pm 3.93$ & $p_{3}<0.001^{*}$ \\
IOP-4 & $17.19 \pm 3.40$ & $p_{4}<0.001^{*}$ \\
$p$-value ${ }^{\dagger}$ & $<0.001$ & \\
CCT $(\mu \mathrm{m})$ & & \\
CCT-b & $555.39 \pm 31.90$ & NA $^{*}$ \\
CCT-1 & $555.00 \pm 30.83$ & $p_{1}=0.191^{*}$ \\
CCT-2 & $555.00 \pm 30.92$ & $p_{2}=0.446^{*}$ \\
CCT-3 & $555.50 \pm 30.83$ & $p_{3}=0.401^{*}$ \\
CCT-4 & $555.00 \pm 31.68$ & $p_{4}=0.704^{*}$ \\
$p$-value & 0.117 & \\
\hline
\end{tabular}

Values are presented as mean \pm standard deviation.

$\mathrm{IOP}=$ intraocular pressure; CCT $=$ central corneal thickness; HBOT $=$ hyperbaric oxygen therapy; NA = not available; $b=$ baseline value; 1 = value after 10 th session of HBOT; $2=$ value after 20th session of HBOT; $3=$ value after 30th session of HBOT; 4 = value after 3 months of last session of HBOT; $p_{1}=$ comparison of baseline and after the 10th session of HBOT; $p_{2}=$ comparison of baseline and after the 20th session of HBOT; $p_{3}=$ comparison of baseline and after the 30th session of HBOT; $p_{4}=$ comparison of baseline and after 3 months of the last session of HBOT.

"Wilcoxon sign rank test, $p<0.05$ was considered statistically significant; "Friedman's ANOVA test, $p<0.01$ was considered statistically significant.

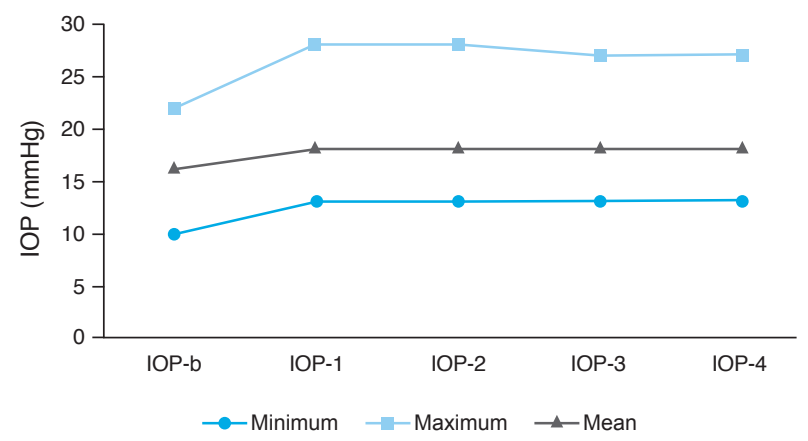

Fig. 1. Intraocular pressure (IOP) changes during hyperbaric oxygen therapy (HBOT). IOP-b = baseline IOP value; IOP-1 = IOP after the 10th session of HBOT; IOP-2 = IOP after the 20th session of HBOT; IOP-3 = after the 30th session of HBOT; IOP-4 $=$ IOP after the three months of the last session of HBOT. 
Table 2. Comparison of RNFL thickness with baseline and consecutive measurement values after HBOT

\begin{tabular}{lcl}
\hline Parameter & Value & $p$-value \\
\hline RNFL $(\mu \mathrm{m})$ & & \\
RNFL-IN-b & $105.86 \pm 30.40$ & NA \\
RNFL-IN-1 & $104.56 \pm 29.24$ & $p_{1}=0.124^{*}$ \\
RNFL-IN-2 & $105.02 \pm 30.63$ & $p_{2}=0.351^{*}$ \\
RNFL-IN-3 & $105.28 \pm 30.33$ & $p_{3}=0.606^{*}$ \\
RNFL-IN-4 & $104.41 \pm 29.39$ & $p_{4}=0.370^{*}$ \\
$p$-value ${ }^{\dagger}$ & 0.096 & \\
RNFL-IT-b & $116.80 \pm 26.46$ & NA \\
RNFL-IT-1 & $117.17 \pm 22.30$ & $p_{1}=0.661^{*}$ \\
RNFL-IT-2 & $118.19 \pm 26.74$ & $p_{2}=0.363^{*}$ \\
RNFL-IT-3 & $117.52 \pm 23.34$ & $p_{3}=0.485^{*}$ \\
RNFL-IT-4 & $116.26 \pm 22.50$ & $p_{4}=0.571^{*}$ \\
$p$-value ${ }^{\dagger}$ & 0.07 & \\
RNFL-SN-b & $100.28 \pm 26.68$ & NA \\
RNFL-SN-1 & $100.60 \pm 25.26$ & $p_{1}=0.697^{*}$ \\
RNFL-SN-2 & $99.47 \pm 27.22$ & $p_{2}=0.477^{*}$ \\
RNFL-SN-3 & $101.41 \pm 26.49$ & $p_{3}=0.289^{*}$ \\
RNFL-SN-4 & $100.95 \pm 26.71$ & $p_{4}=0.455^{*}$ \\
$p$-value ${ }^{\dagger}$ & 0.904 & \\
\hline
\end{tabular}

(Continued)

\begin{tabular}{|c|c|c|}
\hline Parameter & Value & $p$-value \\
\hline RNFL-ST-b & $111.78 \pm 25.04$ & NA \\
\hline RNFL-ST-1 & $112.28 \pm 25.39$ & $p_{1}=0.777^{*}$ \\
\hline RNFL-ST-2 & $112.45 \pm 24.79$ & $p_{2}=0.601^{*}$ \\
\hline RNFL-ST-3 & $112.36 \pm 25.10$ & $p_{3}=0.691^{*}$ \\
\hline RNFL-ST-4 & $111.73 \pm 25.00$ & $p_{4}=0.270^{*}$ \\
\hline$p$-value ${ }^{\dagger}$ & 0.096 & \\
\hline RNFL-T-b & $70.50 \pm 10.13$ & NA \\
\hline RNFL-T-1 & $71.32 \pm 10.28$ & $p_{1}=0.113^{*}$ \\
\hline RNFL-T-2 & $69.56 \pm 8.27$ & $p_{2}=0.313^{*}$ \\
\hline RNFL-T-3 & $70.50 \pm 9.36$ & $p_{3}=0.818^{*}$ \\
\hline RNFL-T-4 & $70.26 \pm 10.28$ & $p_{4}=0.196^{*}$ \\
\hline$p$-value ${ }^{\dagger}$ & 0.02 & \\
\hline RNFL-N-b & $66.71 \pm 11.29$ & NA \\
\hline RNFL-N-1 & $66.63 \pm 10.25$ & $p_{1}=0.882^{*}$ \\
\hline RNFL-N-2 & $66.30 \pm 11.01$ & $p_{2}=0.411^{*}$ \\
\hline RNFL-N-3 & $66.78 \pm 10.34$ & $p_{3}=0.775^{*}$ \\
\hline RNFL-N-4 & $66.45 \pm 10.30$ & $p_{4}=0.237^{*}$ \\
\hline$p$-value ${ }^{\dagger}$ & 0.248 & \\
\hline
\end{tabular}

(Continuing)

Values are presented as mean \pm standard deviation.

$\mathrm{RNFL}=$ retinal nerve fiber layer; HBOT $=$ hyperbaric oxygen therapy; NA = not available; IN $=$ inferonasal; $\mathrm{IT}=$ inferotemporal; $\mathrm{SN}=$ superionasal; $\mathrm{ST}=$ superiotemporal; $\mathrm{N}=$ nasal; $\mathrm{T}=$ temporal; $\mathrm{b}=$ baseline measurement; $1=$ measurements after 10th session of $\mathrm{HBOT}$; $2=$ measurements after 20th session of HBOT; $3=$ measurement after 30th session of HBOT; 4 = measurements after threemonth of last session of HBOT; $p_{1}=$ comparison of baseline and after the 10th session of HBOT; $p_{2}=$ comparison of baseline and after the 20th session of HBOT; $p_{3}=$ comparison of baseline and after the 30th session of HBOT; $p_{4}=$ comparison of baseline and after 3 months of the last session of HBOT.

${ }^{*}$ Wilcoxon sign rank test, $p<0.05$ was considered statistically significant; ${ }^{\dagger}$ Friedman's ANOVA test, $p<0.01$ was considered statistically significant.

Table 3. Comparison of intraocular levels according to the HbAlc values

\begin{tabular}{|c|c|c|c|c|}
\hline \multirow{2}{*}{ IOP value (mmHg) } & \multicolumn{3}{|c|}{ HbAlc (mg/dL) } & \multirow{2}{*}{$p$-value ${ }^{*}$} \\
\hline & $\leq 7.0(\mathrm{n}=17)$ & $7.0<\leq 7.5(\mathrm{n}=17)$ & $>7.5(\mathrm{n}=12)$ & \\
\hline IOP-b & $17(10-21)$ & $16(13-19)$ & $16(14-21)$ & 0.694 \\
\hline IOP-1 & $17(13-22)$ & $17(14-21)$ & $22(14-28)$ & 0.029 \\
\hline IOP-2 & $17(13-22)$ & $17(14-22)$ & $21.5(14-28)$ & 0.018 \\
\hline IOP-3 & $17(13-22)$ & $17(14-22)$ & $22.5(17-27)$ & 0.049 \\
\hline IOP-4 & $16(12-21)$ & $16(14-21)$ & $19(14-21)$ & 0.61 \\
\hline
\end{tabular}

Values are presented as median (min-max).

HbAlc = hemoglobin A1C; IOP = intraocular pressure; $b=$ baseline measurement; 1 = measurements after 10th session of hyperbaric oxygen therapy $(\mathrm{HBOT}) ; 2=$ measurements after 20th session of HBOT; $3=$ measurement after 30th session of HBOT; $4=$ measurements after 3 months of last session of HBOT.

"Kruskal-Wallis test. 
There was significant IOP increases in all measurement times compared to the base-line values $(p<0.05)$ (Table 1 and Fig. 1). During the study period, the IOP level increased over $21 \mathrm{mmHg}$ (between 22 and $28 \mathrm{mmHg}$ ) in seven eyes (15.2\%). These cases were closely followed by whether they had visual field defects with standard automated perimetry 24-2 testing (Humphrey Field Analyzer; Carl Zeiss Meditec, Dublin, CA, USA) once a week until the end of the study. In none of these cases, glaucoma-related visual field defects were observed. These patients no underwent topical antiglaucomatous medication until the end of HBOT. Topical brimonidine tartrate $0.15 \%, 2 \times 1$ was applied into these seven eyes after the last HBOT session. Mean IOP values were higher than baseline values at controls 3 months after the end of HBOT. However, there was no case with IOP above $21 \mathrm{mmHg}$.

No statistically significant difference was observed when CCT values and the six quadrants RNFL were compared with baseline values (Table 1, 2).

At the baseline of the gonioscopic examination, the anterior chamber angles of all the patients were open and slightly pigmentation was present. There was also no difference in the anterior chamber angle examination with the Goldman three-mirror contact lens and optical disc head, peripapillary region, diabetic retinopathy stage in the FFA taken at the end of HBOT compared with initial values. None of the patients required laser or anti-vascular endothelial growth factor treatment during the study period.

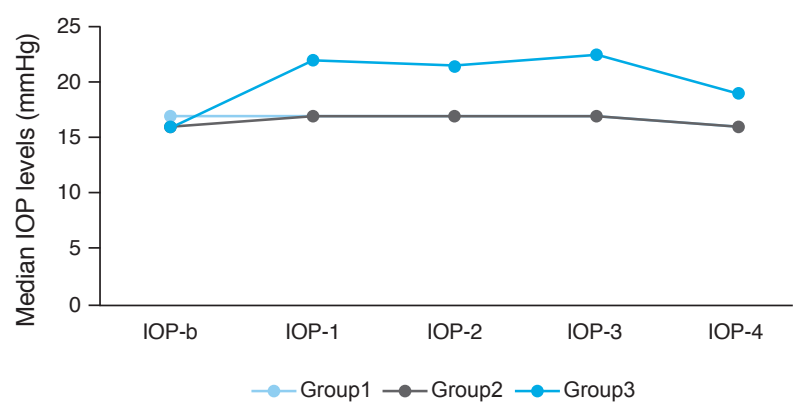

Fig. 2. Median intraocular pressure (IOP) values according to the hemoglobin A1C (HbAlc) levels. IOP-b = baseline IOP value; IOP-1 = IOP after the 10th session of hyperbaric oxygen therapy (HBOT); IOP-2 = IOP after the 20th session of HBOT; IOP-3 = after the 30th session of HBOT; IOP-4 = IOP after the 3 months of the last session of HBOT; Group $1=\mathrm{HbAlc} \leq 7.0 \mathrm{mg} / \mathrm{dL}$ ( $\mathrm{n}=$ 17); Group $2=7.0 \mathrm{mg} / \mathrm{dL}<$ HbAlc $\leq 7.5 \mathrm{mg} / \mathrm{dL}(\mathrm{n}=17)$; Group 3 $=$ HbAlc $>7.5 \mathrm{mg} / \mathrm{dL}(\mathrm{n}=12)$.
HbAlc values were $7.4 \pm 1.9 \%$ in mild-moderated DRP, $8.1 \pm 2.3 \%$ in patients with severe DRP, and $7.3 \pm 1.9 \%$ in patients who had previously received argon laser and inactive DRP. The mean HbAlc values of 7 patients with IOP $>21 \mathrm{mmHg}$ were $8.2 \pm 0.9 \mathrm{mg} / \mathrm{dL}$, and there was significant differences compared with those of patients with IOP values $\leq 21 \mathrm{mmHg}(7.4 \pm 2.8 \mathrm{mg} / \mathrm{dL})(p=0.001)$. There was also significant differences in IOP-1, IOP-2 and IOP-3 values between groups that is created according to the HbAlc levels $(p<0.05)$ (Table 3 and Fig. 2).

\section{Discussion}

For over 50 years HBOT has been regularly used for chronic wound care and yet the underlying mechanisms, clinical effectiveness and side effects are rightly still called into question. When DFU do not heal despite adequate conservative management or progress, HBOT can be considered as an adjuvant therapy [13]. One of crucial effects of HBOT is an increase in angiogenesis activity that leads toformation of new blood vessels and hence increase in nutrients supply to the hypoxic tissue $[14,15]$. There has been a considerable debate on the relationship between HBOT and ROS production in ocular tissues. ROS is associated with several ocular diseases including keratoconus, myopia, cataract, age-related macular degeneration, and glaucoma [7,16-19]. Myopia can be a direct toxic effect of oxygen on the crystalline lens and is the most common side effect of HBOT [16]. Long-term exposures to intensive HBOT were found to be associated with the development of nuclear cataract in support of the theory of associated oxidative damage to the lens proteins [17]. Oxidative stres induce degeneration and death of the retinal pigment epithelium with age-related macular degeneration [18]. For patients who have or are at risk for developing age-related macular degeneration, there may be adverse consequences from increased exposure to ROS associated with HBOT.

Oxidative stress may have a role in the pathogenesis of glaucoma, through degradation of the trabecular network [19]. TM is the most sensitive tissue of the anterior chamber to the oxidative damage. Increased ROS levels and the oxidative stress damage mainly structural and functional components of mtDNA in TM endothelial cells, remodeling of TM cytoarchitecture and this leadsto TM enlargement or collapse [19-21]. Therefore, its oxidative-related 
enlargementor collapse leads to the increased outflow resistance andelevation of IOP [20]. Previous studies have shown that oxidative stress can elevate IOP $[22,23]$. Blood and aqueous humour ROS levels in glaucoma patients were found to be significantly higher than in controls [22]. Analysis of blood plasma samples found that total antioxidant status was decreased in patients with glaucoma $[24,25]$.

There are very few publications in the literature on how IOP fluctuations are affected during HBOT and after the end of treatment. Gallin-Cohen et al. [26] found that HBOT has been associated with a reduction of IOP in rabbits. In other studies conducted by Bojic et al. [27] and Bojic et al. [28], it is showed that HBOT is not significant alterations on IOP in patients with glaucoma. However, Ersanli et al. [29] claimed that IOP was reduced significantly by a mean $1.85 \mathrm{mmHg}$ during HBOT ( 2.5 bar) in their study. In our study, IOP measurements during the study and controls 3 months after the end of the HBOT were statistically higher than the baseline values. Unlike previous studies, the subjects in our study were patients without glaucoma before HBOT and all with type 2 DM. The IOP increase in our patients may be due to HBOT or the additional contribution of type $2 \mathrm{DM}$. Larger clinical studies evaluating the effect of future HBOT on IOP will clarify this problem.

In our study, when examined IOP changes of 46 patients with type $2 \mathrm{DM}$ who were treated with HBOT for 30 sessions with diabetic foot ulser diagnosis, IOP measurements of seven $(15.2 \%)$ eyes after the 10th session of treatment were above $21 \mathrm{mmHg}$ (between 22 and $28 \mathrm{mmHg}$ ). IOP measurements of these subjects were also above $21 \mathrm{mmHg}$ during HBOT. After the last session HBOT, topical anti-glaucomatous drops were started in these eyes. When these patients were compared with those of IOP $\leq 21$ $\mathrm{mmHg}$, their HbAlc values were higher than those of patients with IOP values $\leq 21 \mathrm{mmHg}$. This result shows that poor blood glucose regulation may cause IOP elevation in HBOT cases. Hyperglycemia and high HbAlc levels lead to free radical production by glucose oxidation, non-enzymatic glycation of proteins and elevation of lipid peroxidation $[30,31]$. Exaggerated ROS production triggering by hyperglycemia can reduce the antioxidant enzyme activities directly. Both free radical formation by HBOT and poor blood glucose regulation may exceed the neutralizing capacity of antioxidant systems of the eye, and HBOT-re- lated side effects may increase in these patients.

The use of antioxidants is critical to combat the oxidative stressors caused by the production of ROS and for the maintenance of homeostasis. Under conditions of ROS overproduction, supplementation with compounds of a high antioxidant potential is immensely valuable. The use of antioxidant compounds in the treatment of glaucoma have been previously studied. Some previous animal and human studies found that antioxidant supplementation can improve insulin resistance and decrease blood glucose concentrations and HbAlc [32-34]. On the other hand, vitamin E, N-acetylcysteine, and resveratrol have previously been shown to be beneficial in the management of primer open glaucoma $[35,36]$. Furthermore, the consumption of flavonoid containing foods has been found to reduce the overall risk of primer open glaucoma and benefit longterm visual function [37]. Hodges et al. [38] found that intravenously administration of $1 \mathrm{mg} / \mathrm{kg}$ myricetin decreased IOP in normotensive rabbits. Based on these studies, antioxidant supplementation may be considered to prevent ocular side effects such as IOP increase due to ROS in DFU patients receiving HBOT treatment.

As it is known, CCT values affect IOP measurements. We not observed any significant differences in CCT values during our study compared with baseline values. Similar findings were found in the study of Ayata et al. [39]. The study measured CCT with an ultrasonic pachymeter before and immediately after HBO treatment, which lasted 120 minutes at 2.4 atmospheres absolute with three $30-\mathrm{min}$ oxygen and two 5-minute air breathing periods. They foud that HBO treatment did not change the CCT in diabetic subjects, however, the CCT was significantly reduced after HBO treatment in nondiabetic subjects. Our study was different than the Ayata et al. [39] study that was containing a non-diabetic control group, but our study did not include non-diabetic patients.

In our study, we also detected that although there was a significant increase in IOP, no change was detected in RNFL examination. This result suggested that HBOT may have a positive effect on the optic nerve despite the increase in IOP. Although there is not any study evaluating the effect of HBOT on RNFL in the literature, some case series investigating the efficacy of HBOT in optic nerve diseases have been reported. In one of these series, HBOT administered in four cases due toradiation-induced optic neuropathy. According to this study, HBOT was useful in 
two patients who have received HBOT within 72 hours after visual loss, however, it was not any benefit in the other two patients who have treated with $\mathrm{HBO}$ two weeks after visual loss [40]. Conversely, in another study, the visual acuity improved in a patient who have treated with HBO 17 weeks after the onset of radiation-induced optic neuropathy [41].

HBOT provided significant improvement of visual acuity and field in patients with non-arteritic anterior ischemic optic neuropathy [42]. HBOT also temporarily improves visual symptoms in patients with multiple sclerosis [43]. Nevertheless, HBOT can not be recommended in these patients until more advanced and well-designed studies are reported.

One of the two important results we obtained in our study is that HBOT may increase IOP, especially in patients with impaired blood glucose regulation. For this reason, IOP follow-up should be strictly performed in patients with poor blood glucose levels, and supplementation with an anti-oxidant agent may be considered as an option in these cases. Another result is not observed any changes in RNFL thichness in spite of increase in IOP values.

One of the main limitations of the study is that the effect of HBOT on IOP change in individuals without diabetes could not be investigated. Secondly, we have not demonstrated the histopathologic effects of HBOT on ocular tissue samples such as trabeculum that is more accurate approach to evaluate the results of this study, however; this is only possible by examining animal experiments and/or dead human tissue specimens.

In the light of our study results, studies involving ROS measurements in anterior chamber samples can be performed to find the reasons for IOP increase in HBOT treatment. In addition, the effects of antioxidant therapy on IOP may be investigated in diabetic patients with poor blood glucose regulation during HBOT.

\section{Conflict of Interest}

No potential conflict of interest relevant to this article was reported.

\section{References}

1. Kaur S, Pawar M, Banerjee N, Garg R. Evaluation of the efficacy of hyperbaric oxygen therapy in the management of chronic nonhealing ulcer and role of periwound transcutaneous oximetry as a predictor of wound healing response: a randomized prospective controlled trial. J Anaesthesiol Clin Pharmacol 2012;28:70-5.

2. Oter S, Korkmaz A, Topal T, et al. Correlation between hyperbaric oxygen exposure pressures and oxidative parameters in rat lung, brain, and erythrocytes. Clin Biochem 2005;38:706-11.

3. Benedetti S, Lamorgese A, Piersantelli M, et al. Oxidative stress and antioxidant status in patients undergoing prolonged exposure to hyperbaric oxygen. Clin Biochem 2004;37:312-7.

4. Dennog C, Radermacher P, Barnett YA, Speit G. Antioxidant status in humans after exposure to hyperbaric oxygen. Mutat Res 1999;428:83-9.

5. Palmquist BM, Philipson B, Barr PO. Nuclear cataract and myopia during hyperbaric oxygen therapy. $\mathrm{Br} J$ Ophthalmol 1984;68:113-7.

6. Tokarz P, Kaarniranta K, Blasiak J. Role of antioxidant enzymes and small molecular weight antioxidants in the pathogenesis of age-related macular degeneration (AMD). Biogerontology 2013;14:461-82.

7. Shoham A, Hadziahmetovic M, Dunaief JL, et al. Oxidative stress in diseases of the human cornea. Free Radic Biol Med 2008;45:1047-55.

8. Arnal E, Peris-Martinez C, Menezo JL, et al. Oxidative stress in keratoconus? Invest Ophthalmol Vis Sci 2011;52:8592-7.

9. Mozaffarieh M, Grieshaber MC, Flammer J. Oxygen and blood flow: players in the pathogenesis of glaucoma. Mol Vis 2008;14:224-33.

10. Ferreira SM, Lerner SF, Brunzini R, et al. Antioxidant status in the aqueous humour of patients with glaucoma associated with exfoliation syndrome. Eye (Lond) 2009;23:16917.

11. Zhou L, Li Y, Yue BY. Oxidative stress affects cytoskeletal structure and cell-matrix interactions in cells from an ocular tissue: the trabecular meshwork. J Cell Physiol 1999;180:182-9.

12. Li AF, Tane N, Roy S. Fibronectin overexpression inhibits trabecular meshwork cell monolayer permeability. Mol Vis 2004;10:750-7.

13. Londahl M. Hyperbaric oxygen therapy as adjunctive 
treatment of diabetic foot ulcers. Med Clin North Am 2013;97:957-80.

14. Johnston BR, Ha AY, Brea B, Liu PY. The mechanism of hyperbaric oxygen therapy in the treatment of chronic wounds and diabetic foot ulcers. R I Med J (2013) 2016;99:26-9.

15. Stoekenbroek RM, Santema TB, Legemate DA, et al. Hyperbaric oxygen for the treatment of diabetic foot ulcers: a systematic review. Eur J Vasc Endovasc Surg 2014;47:64755.

16. Evanger $\mathrm{K}$, Haugen $\mathrm{OH}$, Irgens A, et al. Ocular refractive changes in patients receiving hyperbaric oxygen administered by oronasal mask or hood. Acta Ophthalmol Scand 2004;82:449-53.

17. Plafki C, Peters $\mathrm{P}$, Almeling $\mathrm{M}$, et al. Complications and side effects of hyperbaric oxygen therapy. Aviat Space Environ Med 2000;71:119-24.

18. Friedman DS, O'Colmain BJ, Munoz B, et al. Prevalence of age-related macular degeneration in the United States. Arch Ophthalmol 2004;122:564-72.

19. Sacca SC, Izzotti A, Rossi P, Traverso C. Glaucomatous outflow pathway and oxidative stress. Exp Eye Res 2007;84:389-99.

20. Chrysostomou V, Rezania F, Trounce IA, Crowston JG. Oxidative stress and mitochondrial dysfunction in glaucoma. Curr Opin Pharmacol 2013;13:12-5.

21. Izzotti A, Sacca SC, Longobardi M, Cartiglia C. Mitochondrial damage in the trabecular meshwork of patients with glaucoma. Arch Ophthalmol 2010;128:724-30.

22. Caballero M, Liton PB, Epstein DL, Gonzalez P. Proteasome inhibition by chronic oxidative stress in human trabecular meshwork cells. Biochem Biophys Res Commun 2003;308:346-52.

23. Fuchshofer R, Yu AH, Welge-Lussen U, Tamm ER. Bone morphogenetic protein-7 is an antagonist of transforming growth factor-beta2 in human trabecular meshwork cells. Invest Ophthalmol Vis Sci 2007;48:715-26.

24. Nucci C, Di Pierro D, Varesi C, et al. Increased malondialdehyde concentration and reduced total antioxidant capacity in aqueous humor and blood samples from patients with glaucoma. Mol Vis 2013;19:1841-6.

25. Mousa A, Kondkar AA, Al-Obeidan SA, et al. Association of total antioxidants level with glaucoma type and severity. Saudi Med J 2015;36:671-7.

26. Gallin-Cohen PF, Podos SM, Yablonski ME. Oxygen lowers intraocular pressure. Invest Ophthalmol Vis Sci
1980;19:43-8.

27. Bojic L, Kovacevic H, Andric D, et al. Hyperbaric oxygen dose of choice in the treatment of glaucoma. Arh Hig Rada Toksikol 1993;44:239-47.

28. Bojic L, Racic G, Gosovic S, Kovacevic H. The effect of hyperbaric oxygen breathing on the visual field in glaucoma. Acta Ophthalmol (Copenh) 1993;71:315-9.

29. Ersanli D, Akin T, Yildiz S, et al. The effect of hyperbaric oxygen on intraocular pressure. Undersea Hyperb Med 2006;33:1-4.

30. Maritim AC, Sanders RA, Watkins JB 3rd. Diabetes, oxidative stress, and antioxidants: a review. J Biochem Mol Toxicol 2003;17:24-38.

31. Matough FA, Budin SB, Hamid ZA, et al. The role of oxidative stress and antioxidants in diabetic complications. Sultan Qaboos Univ Med J 2012;12:5-18.

32. Vafa M, Mohammadi F, Shidfar F, et al. Effects of cinnamon consumption on glycemic status, lipid profile and body composition in type 2 diabetic patients. Int $J$ Prev Med 2012;3:531-6.

33. Opara EC. Role of oxidative stress in the etiology of type 2 diabetes and the effect of antioxidant supplementation on glycemic control. J Investig Med 2004;52:19-23.

34. Al-Maskari MY, Waly MI, Ali A, et al. Folate and vitamin B12 deficiency and hyperhomocysteinemia promote oxidative stress in adult type 2 diabetes. Nutrition 2012;28:e23-6.

35. Ko ML, Peng PH, Hsu SY, Chen CF. Dietary deficiency of vitamin $\mathrm{E}$ aggravates retinal ganglion cell death in experimental glaucoma of rats. Curr Eye Res 2010;35:842-9.

36. Ramdas WD, Wolfs RC, Kiefte-de Jong JC, et al. Nutrient intake and risk of open-angle glaucoma: the Rotterdam Study. Eur J Epidemiol 2012;27:385-93.

37. Patel S, Mathan JJ, Vaghefi E, Braakhuis AJ. The effect of flavonoids on visual function in patients with glaucoma or ocular hypertension: a systematic review and meta-analysis. Graefes Arch Clin Exp Ophthalmol 2015;253:1841-50.

38. Hodges LC, Kearse CE, Green K. Intraocular pressure-lowering activity of phenolic antioxidants in normotensive rabbits. Curr Eye Res 1999;19:234-40.

39. Ayata A, Uzun G, Mutluoglu M, et al. Influence of hyperbaric oxygen therapy on central corneal thickness. Ophthalmic Res 2012;47:19-22.

40. Guy J, Schatz NJ. Hyperbaric oxygen in the treatment of radiation-induced optic neuropathy. Ophthalmology 1986;93:1083-8.

41. Borruat FX, Schatz NJ, Glaser JS, et al. Visual recovery 
from radiation-induced optic neuropathy. The role of hyperbaric oxygen therapy. J Clin Neuroophthalmol 1993;13:98-101.

42. Bojic L, Ivanisevic M, Gosovic G. Hyperbaric oxygen therapy in two patients with non-arteritic anterior optic neu- ropathy who did not respond to prednisone. Undersea Hyperb Med 2002;29:86-92.

43. Bennett M, Heard R. Hyperbaric oxygen therapy for multiple sclerosis. Cochrane Database Syst Rev 2004;(1):CD003057. 